\title{
The Use of Image Processing and Analysis in Automated Biological Dosimetry
}

\author{
D. Ramadhani ${ }^{1,2 *}$, M. Syaifudin ${ }^{2}$, S. Purnami ${ }^{2}$ and A. Naroeni ${ }^{3}$ \\ ${ }^{I}$ Doctoral Program for Biomedical Sciences, Faculty of Medicine, Universitas Indonesia (UI), \\ Jl. Salemba Raya 6, Jakarta 10430, Indonesia \\ ${ }^{2}$ Center for Technology of Safety and Radiation Metrology, National Nuclear Energy Agency (BATAN), \\ Jl. Lebak Bulus Raya No. 49, Jakarta 12070, Indonesia \\ ${ }^{3}$ Virology and Cancer Pathobiology Research Center, Faculty of Medicine, Universitas Indonesia (UI), \\ Jl. Salemba Raya 4, Jakarta 10430, Indonesia
}

\section{ARTICLE INFO}

Article history:

Received 14 June 2019

Received in revised form 22 September 2020 Accepted 6 October 2020

Keywords:

Biodosimetry

Dicentric chromosome

$\gamma-\mathrm{H} 2 \mathrm{AX}$

Image analysis

Micronuclei

\begin{abstract}
A B S T R A C T
Biological dosimetry based on cytogenetic markers such as dicentric chromosome (DC) and micronuclei (MN) is, until now, the most frequently used method to estimate the radiation dose in the radiological accident event. Another biomarker that recently gains popularity in biodosimetry is $\gamma \mathrm{H} 2 \mathrm{AX}$. All these three assays are microscope-based biodosimetry techniques, and therefore need manual scoring to estimate the radiation dose. Unfortunately, the manual scoring of these assays is time-consuming and labor-intensive. In the case of a large-scale radiological accident when many people are exposed to radiation, it is very useful to use image processing and analysis in the scoring process to obtain a faster result. Several commercial systems or open-source image processing software packages already developed automated scoring of DC, MN, and $\gamma \mathrm{H} 2 \mathrm{AX}$ assays. This article describes how image processing and analysis were applied in automated biodosimetry based on the $\mathrm{DC}, \mathrm{MN}$, and $\gamma \mathrm{H} 2 \mathrm{AX}$ assays.
\end{abstract}

(C) 2020 Atom Indonesia. All rights reserved

\section{INTRODUCTION}

Nowadays, exposure to ionizing radiation during human life is inevitable, and the risk of exposure has increased due to the wide application of radiation-producing technologies, including energy sources, medical therapy, and manufacturing processes. Nuclear energy is considered as an important energy source in several countries $[1,2]$ However, the increase in the number of nuclear facilities worldwide has concomitantly increased the probability of radiation accidents. Moreover, during large-scale radiological exposures to the public, caused by accidental events at nuclear facilities, the high number of individuals that could potentially be exposed to varying doses of radiation should be considered. In this case, the assessment of radiation doses received by exposed persons should be conducted as soon as possible and with a high level of accuracy. The prediction of biological effects is

\footnotetext{
*Corresponding author.

E-mail address: dhani02@batan.go.id

DOI: https://doi.org/10.17146/aij.2020.960
}

also important to facilitate the selection of the correct medical treatments for victims [3]. In the event of radiological accidents, when a personal physical dosimeter is unavailable, estimations regarding the absorbed radiation doses received by the public can only be performed using biological dosimeters $[1,2,4]$.

Biological dosimetry (biodosimetry) is defined as the use of biological endpoints as biomarkers of radiation exposure during radiation dose prediction [5]. During the last decade, the use of "omics" markers in biodosimetry has gained popularity among many radiobiological investigators. However, the use of these markers in biodosimetry has been limited because none of these biomarkers have been tested or validated during real radiological accidents to obtain reliable dose estimations. Furthermore, "omics" markers are more suitable as bioindicators of radiation exposure rather than biodosimeters due to the high inter-individual variations among their baseline frequencies [1]. Over the past decades, the most commonly used biomarkers for biodosimetry has been cytogenetic 
markers. Among the many cytogenetic endpoints used for biodosimetry, the dicentric chromosome assay (DCA) has been the most widely used method until now [4]. In DCA, the frequencies of dicentric chromosomes in mitotic lymphocytes are counted and then converted into an estimation of the ionizing radiation dose [3]. The DCA is considered as the gold standard for biodosimetry, due to its low background, high comparability between the in vivo and in vitro dose responses, and low inter-individual variability. This assay could also be used to differentiate between partial and whole-body exposure, as well as between high- and low-linear energy transfer (LET) exposure [6]. Cytogenetic biodosimetry has already been used to estimate the radiation doses received by individuals in several nuclear accidents. It was used on Chernobyl accident victims, and it was also used on the victims of the accident at Fukushima, when a massive earthquake and a subsequent tsunami in the northeastern area of Japan caused the release of a significant amount of radioactive materials into the environment in 2011 [7].

Although DCA has been shown to provide an accurate estimation of radioactive doses, the process is laborious and time-consuming. Thus, in the event of large-scale radiological or nuclear accidents involving hundreds or thousands of individuals, triage versions of DCA that increase the speed of sample processing and dose estimation are necessary [8]. Another promising technique that can overcome the sample processing speed limitations of DCA is the use of image processing and analysis to automate dicentric scoring during the DCA [9]. Image processing and analysis have been used to automate not only the DCA but also the cytokinesis-block micronucleus (CBMN) assay. The CBMN assay is one of the most commonly used traditional cytogenetic biodosimetry techniques, in addition to DCA. In the CBMN assay, the frequency of micronuclei $(\mathrm{MN})$ inside binucleated cells (BNCs) can be used to extrapolate the dose of ionizing radiation. Similar to DCA, CBMN assay has also been standardized for biodosimetry using manual microscopy. However, compared with DCA, CBMN assay is less specific, and background levels of $\mathrm{MN}$ can be affected by age, gender, and such lifestyle factors as smoking and diet [10].

$\mathrm{H} 2 \mathrm{~A}$ histone family member $\mathrm{X}(\mathrm{H} 2 \mathrm{AX})$ is a histone that becomes phosphorylated $(\gamma \mathrm{H} 2 \mathrm{AX})$ at the site of DNA double-strand breaks (DSBs), and the presence of one $\gamma \mathrm{H} 2 \mathrm{AX}$ focus represent one DSB in DNA. Recently, the presence of $\gamma \mathrm{H} 2 \mathrm{AX}$ has also frequently been used as a biomarker for radiation exposure. The number of $\gamma \mathrm{H} 2 \mathrm{AX}$ foci also increases linearly with increasing radiation doses
[10]. The quantification of $\gamma \mathrm{H} 2 \mathrm{AX}$ foci can be performed manually, by counting the number of foci, or automatically, by evaluating $\gamma \mathrm{H} 2 \mathrm{AX}$ immunofluorescence intensity using recent technology, such as flow cytometry. Unfortunately, the manual quantification method is considered to be time-consuming, and foci interpretation can be subjective. In contrast, the use of flow cytometry can provide faster results but is less sensitive compared with manual quantification; thus, this method is not suitable for low-dose radiation damage assessments. In this case, image processing and analysis could be used for pattern recognition, to facilitate the counting and size calculations of foci [14]. Because many open-source software options are now available for biological or biomedical image processing and analysis, the implementation of image analysis during biodosimetry could easily be performed to obtain faster results. This article will describe how the image processing and analysis have been applied to automated biodosimetry methods based on DC, CBMN, and $\gamma \mathrm{H} 2 \mathrm{AX}$ assays. In this article, only studies published after 2013 are described, to provide the newest information regarding the implementation of image processing and analysis for DC, CBMN, and $\gamma \mathrm{H} 2 \mathrm{AX}$ assays.

\section{APPLICATIONS OF IMAGE PROCESSING AND ANALYSIS IN AUTOMATED BIODOSIMETRY}

\section{Dicentric chromosome assay}

The use of image processing and analysis and software development to automatically identify DC chromosomes has emerged over the last several years. Interestingly, the implementation of image processing and analysis in the DCA possess has presented greater challenges than the implementation of the analysis in the $\mathrm{MN}$ and $\gamma \mathrm{H} 2 \mathrm{AX}$ assays. Variability among the morphologies of metaphase chromosomes and differences among protocol methods across different laboratories have resulted in the inconsistent appearance of chromosomes. The presence of overlapping or touching chromosomes also increases the difficulty of DC detection using image analysis [11]. Currently, the most powerful commercial package for biodosimetry DCA is Metafer, from MetaSystems (Altlussheim, Germany). This system consists of software modules for metaphase identification (MSearch), the auto-capture of high-resolution images at $63 \times$ magnification (AutoCapt), and the automatic detection of dicentric chromosomes (DCScore) [12]. Several laboratories 
have used the DCScore module in Metafer to perform semi-automated DC scoring, to increase the rapidity of DC analysis [13].

In 2018, Dai et al. [14] proposed the 3 -gradient scanning method, based on three different search windows for automatic metaphase cell identification, as illustrated in Fig. 1. In their study, Dai et al. used three search windows for each slide: $5 \%$ proximal area, $35 \%$ central area, and $50 \%$ distal area. They claimed that using this concept increased the analysis speed without affecting the accuracy of dose estimation. During conventional automatic chromosome scanning and analysis using the Metafer system, the process of scanning and obtaining high-resolution digital images of chromosomes can be time-consuming. Using the 3 -gradient scanning method, the chromosome scanning and analysis speed was improved, and the number of photographed images necessary to obtain estimated radiation doses were reduced [14].

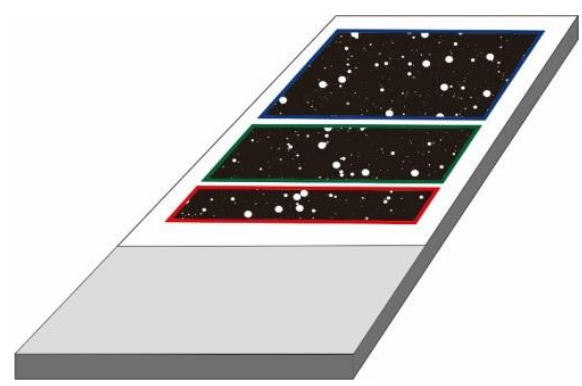

Fig. 1. The three setting regions the searching window in 3 -gradient scanning method. The red square represented $5 \%$ proximal area, whereas the $35 \%$ central area was represented in blue square. Last the $50 \%$ distal area of slide, was in the blue square. Figure was redrawn, based on Dai et al. [14].

Although DCScore has been used in many laboratories, the manual supervision of DC analysis remains necessary when using this software. The high rate of false-positive (FP) DC identifications using DCScore could be due to variations among chromosome morphologies. Chromosome quality is a key determining factor for the success of automated or semi-automated scoring systems. When high-quality metaphase chromosomes are used, with no overlapping chromosomes or staining debris, the automated detection of DCs is highly accurate (> $92-95 \%$ of manual scoring) [13]. Another piece of software that has been developed for the automatic identification of DCs is Automated Dicentric Chromosome Identifier (ADCI), by Rogan group, which was initially released in 2010 [11,15-17]. The ADCI software uses image segmentation techniques to extract objects that might be considered chromosomes. Image filtering during a preprocessing step removes most, but not all, non-chromosomal objects, such as debris, nuclei, and overlapping chromosomes. The remaining objects are then regarded as "chromosome-like" objects. Each object is then processed by a series of algorithms, which create a quantitative profile that measures chromosome width from one telomere to the other. Centromere candidates or potential centromere locations are then identified at constrictions in the width profile [16]. Unfortunately, obtaining metaphase images still requires the AutoCapt module from MetaSystems; therefore, this software package is not fully independent from other commercial capturing systems.

\section{CBMN assay}

The implementation of image processing and analysis for CBMN assay began three decades ago. The CBMN assay is one of the preferred techniques for assessing chromosomal damage induced by mutagens, such as radiation exposure, in human populations [18]. Several automated CBMN assay systems have previously been tested (i.e., Metafer Metasystem, PathFinder, CellScan, imaging flow cytometry) to determine whether these systems can be used during large-scale radiological events [18]. The detection of $\mathrm{MN}$ and BNCs during CBMN assay can be performed using several different thresholding levels, using either an image stained with a single dye that labels both nuclei and whole cells, such as Giemsa, or using an image where the cytoplasm has been counterstained using a specific dye [19]. However, the use of Giemsa staining can cause several problems, such as the appearance of debris that can be misidentified as $\mathrm{MN}$; therefore, this staining protocol is not considered suitable for automated processes. A more common stain used during automated processes is 4',6-diamidino-2phenylindole (DAPI), which only stains the nuclei. A secondary staining to identify the boundaries of each cell can also be performed, although this step is often omitted, likely because it significantly reduces throughput. More commonly, MN associated with the nucleus are predicted using the proximity method [20].

A research group from the Center for High-Throughput Minimally Invasive Radiation Biodosimetry at Columbia University has developed a robotic platform called Robotic Automated Biodosimetry Technology (RABiT) for the highthroughput processing of cytogenetic biodosimetry techniques, such as the CBMN assay and DCA $[19,20]$. In several of their recent publications, this group has described the development of RABiT-II, with the aim to implement standard cytogenetic 
assays in a 96-well plate format and analyzing them using commercial, automated, high-throughput screening platforms. In 2019, Repin et al. proposed a new concept, combining RABiT-II with the IN Cell Analyzer 2000 and fully-automated image analysis (GE Healthcare IN Cell Developer Toolbox). They also reduced the culture time to $54 \mathrm{~h}$, which is much shorter than the 68-74 h required for classical CBMN assays. A shorter culture time would significantly enhance the utility of CBMN assays during large-scale radiological events [21]. In this study, they also used standard-height, 96-well, $450-\mu 1$ volume microplates for the fixation of cultures after the incubation process. For the identification of BNCs, they used the following criteria. BNCs should consist of two nuclei, with a combined area greater than $70 \mu^{2}$, with similar characteristics, and separated by a distance less than three times their average radius. BNC-associated nuclei were scored as $\mathrm{MN}$ if the distance from the center of each micronucleus to the midpoint between the centers of the two nuclei in the associated BNC was less than $15 \mu \mathrm{m}$ and if the MN area was less than one third of the average area of the two nuclei in the associated BNC, as illustrated in Fig. 2. In total, this process required approximately $61 \mathrm{~h}$, including $54 \mathrm{~h}$ for incubation, $1 \mathrm{~h}$ for the transfer process to a 96-well plate, and $6 \mathrm{~h}$ for automated image analysis to evaluate 96 samples [21]. The total time necessary for the combination of the RABiT-II system, the IN Cell Analyzer 2000, and the fullyautomated image analysis was less than the total time necessary to analyze 96 samples using the conventional method.

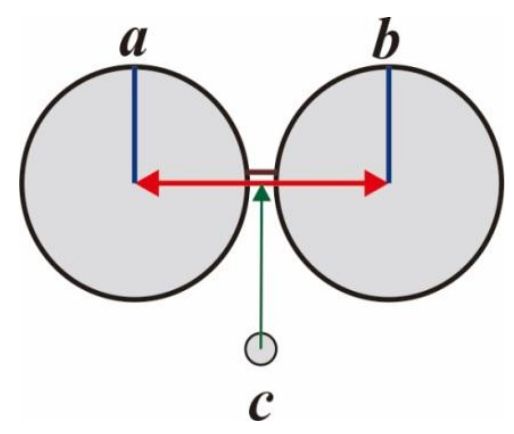

Fig. 2. BNC and MN identification criteria in Repin et al. study $a$ and $b$ are the two nuclei, whereas $c$ is the MN. The total area of $a$ or $b$ should be more than $70 \mu \mathrm{m}^{2}$. The distance between $a$ and $b$ (brown line) should less than $3 \times$ of $a$ and $b$ average radius (blue lines). The distance of MN center to the midpoint between the centers of two nuclei in the associated BNC (green line) should less than $15 \mu \mathrm{m}$. MN area should less than one-third of the average area of the two nuclei ( $a$ and $b$ ). Figure was drawn, based on Repin et al. study [21].

François et al., in 2014, used laser scanning cytometry (LSC) technology to automatically measure MN [18]. The LSC protocol consisted of several steps. First, the areas where the cells were cytocentrifuged onto the slide were scanned using an automated LSC protocol. Each cytospot contained a minimum of 1000 cells. After the scanning process was completed, the numbers of mononucleated, binucleated, and multinucleated (> 2 nuclei) cells and the numbers of $\mathrm{MN}$ inside the BNCs were retrieved. In detail, the LSC system used specific contours around the cytoplasm, nuclei, and MN to allow the identification of cells associated with MN. This protocol, as illustrated in Fig. 3, allowed automatic contours to be drawn surrounding the cytoplasm (green), nuclei (yellow), and $\mathrm{MN}$ (magenta). The separation of BNCs from the rest of the cell population and the subsequent scoring of MN was also possible using this protocol [18]. Based on prior experience, open-source cell imaging analysis software packages, such as CellProfiler, can be used for the automatic detection of BNCs and MN [22]. The "parent and child relationship" concept between cells and nuclei utilized in CellProfiler can be used to identify BNCs. By considering cells as parents, it should be possible to identify BNCs that have two nuclei (children). Cells are not counted as BNCs if they have only one or more than two nuclei [23]. This concept can be especially effective when used on images that use specific dyes for the cytoplasm and the nucleus, such as those described by François et al. [18]. Overall, during the automation of the CBMN assay, reducing the culture time should also be considered to achieve faster results.

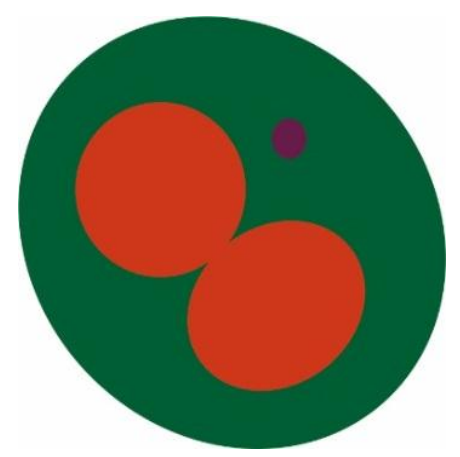

Fig. 3. Illustration of BNC containing MN from François et al. study [18].

\section{$\gamma \mathrm{H} 2 \mathrm{AX}$}

The $\gamma \mathrm{H} 2 \mathrm{AX}$, which can be detected by immunostaining, is a surrogate marker for radiationinduced DNA DSBs [24]. The quantification of foci is generally performed manually, introducing the potential for inconsistencies and human errors [25]. Manual analysis also results in high variability among observers. Frequently, the number of foci identified in the first image analyzed becomes the ground truth for all subsequent images and other related studies. Therefore, the use of image 
processing and analysis for foci detection could overcome the limitations observed for manual analysis. Herbert et al., in 2014, developed a machine learning system based on open-source software package ImageJ, called FindFoci, which operates as an ImageJ plugin. For FindFoci to function, it requires the operator to first train the algorithm to closely match the focus assignment, using several images, and then applying those parameters across a large number of images. FindFoci also facilitates transparency among the parameters used by another operator to detect foci and provides visual tools that can be used to compare different experimenters' detections of foci [25].

In 2015, Lapytsko et al. developed an opensource, user-friendly software program with a graphical user interface (GUI) for individual foci counting, called FoCo. Although FoCo is opensource, it was developed in Matlab, using the Image Processing Toolbox (IPT), which is a commercial software package [26]. Another opensource software package for the automated quantitative and qualitative analysis of foci is Focinator, which was developed as a macro for ImageJ [27]. Although several macros for foci counting had previously been developed, Focinator provided an adjustable and user-friendly macro based on ImageJ that could be used for quantitative and qualitative analysis of nuclei, $\gamma \mathrm{H} 2 \mathrm{AX}$ foci, and other biological foci, facilitating easy data export and processing. Using Focinator, the analysis time for processing 35 multi-channel images was significantly reduced by approximately 23 -fold, taking only $5.61 \mathrm{~min}$ with Focinator, which was much faster than the 132.07 min required for manual analysis. Focinator facilitates the automated detection of foci and the analysis of regions of interest (ROIs) and foci. When using Focinator, selected ROIs can be measured, including the total area and the mean, minimal, and maximal grey values within the selection. The foci can then be detected based on the "Find maxima" command in ImageJ. Using this command, ImageJ identifies signal peaks within the 16-bit grey scale of an image compared with the greyscale values of the surrounding pixels [27].

Focinator was refined in 2017 to become Focinator v2.0 [28]. In the new version of Focinator, the software becomes more user-friendly than the original version released in 2015 , due to a new GUI. Focinator v2.0 is also able to count and analyze foci in up to three channels. Focinator can be run in full-automated mode or semi-automated mode, using an R-script-based mode. Although Focinator can be very powerful for automated foci analysis, it may have difficulties analyzing three-dimensional (3D) or multilayer images. Until now, most 3D analyses have been performed using commercial software packages, such as Imaris (Bitplane AG). Ruprecht et al., in 2018, used Imaris to perform 3D reconstructions to improve the detection and analysis of $\gamma \mathrm{H} 2 \mathrm{AX}$ fluorescence signals [29]. Ruprecht et al. decided to perform 3D reconstructions since the $3 \mathrm{D}$ nature of the nucleus is rarely considered during conventional fluorescence microscopy-based $\gamma \mathrm{H} 2 \mathrm{AX}$ foci analysis. During the visualization of $\gamma \mathrm{H} 2 \mathrm{AX}$ foci using fluorescence microscopy, the entirety of the fluorescence signal is visualized, without the clear delineation of individual foci. Thus, by using a confocal microscope with 3D imaging, a more realistic determination of the numbers, sizes, and spatial arrangements of $\gamma \mathrm{H} 2 \mathrm{AX}$ foci can be determined. The comparisons between foci per cell values obtained using 3D reconstructed images and those obtained using major intensity projection and conventional fluorescence microscope techniques showed significant differences among the predicted doses, except in the non-irradiated samples, as presented in Fig. 4. Overall, the automated $\gamma \mathrm{H} 2 \mathrm{AX}$ assay using image processing and analysis has been shown to be much better than manual analysis, because manual analysis cannot provide such additional information as the size of the nuclei or the intensity of the foci [29].

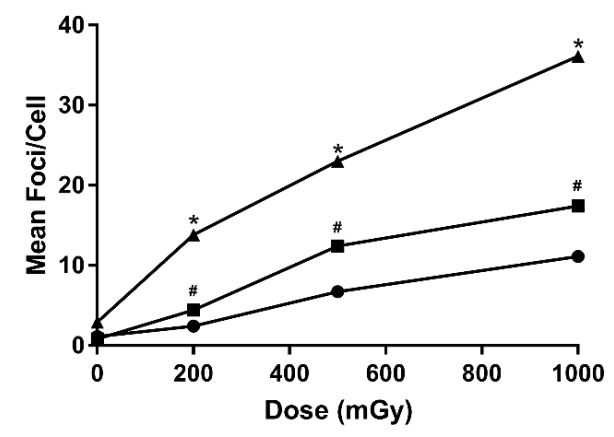

Fig. 4. Comparison of $\gamma \mathrm{H} 2 \mathrm{AX}$ foci/cell values derived by conventional fluorescence microscope $(\mathrm{CFM})(\bullet)$, confocal microscope with maximum intensity projection (MIP) (๘), and confocal microscope with $3 \mathrm{D}$ reconstruction $(3 \mathrm{DR})(\mathbf{\Delta})$. *:Significantly different compared to CFM, \#:Significantly different compared to 3DR. Figure were drawn, based on Ruprecht et al. study [29].

\section{CURRENT STATUS OF AUTOMATIZATION OF BIODOSIMETRY USING IMAGE PROCESSING AND ANALYSIS IN INDONESIA}

The National Biodosimetry Laboratory in Indonesia was established in 2005 by the National Nuclear Energy Agency of Indonesia, known locally as Badan Tenaga Nuklir Nasional (BATAN). Our research group within BATAN established the 
national standard dose-response calibration curves for DCA and MN in 2013, 2016, 2017, and 2019 [30-34]. The establishment of a national standard dose-response calibration curve for $\gamma \mathrm{H} 2 \mathrm{AX}$ is currently in progress in the laboratory. In 2015, the biodosimetry laboratory purchased a Metasystems Metafer3 Scanning System, mounted on a Zeiss Axio Imager Z2 microscope. However, the aforesaid Metafer3 System consists of the AutoCapt module only, without the DCScore module. Thus, the Metafer3 System is used only to find and automatically capture the metaphase chromosomes, without using the DCScore module to automatically recognize and score the DC. Since the use of the DCScore module could dramatically increase the speed of the DCA, provision of the DCScore module for the Metafer3 system would be advantageous. However, an initial study needs to be conducted to obtain high-quality metaphase chromosomes, to avoid the high rate of false-positive DC identification using the DCScore module. An opensource image processing and analysis software package (CellProfiler) was used to automatically identify BNCs and MN in 2013 by our research group [23]. However, high levels of debris appeared during analysis using CellProfiler, and the debris was misclassified as $\mathrm{MN}$ due to the use of Giemsa staining. Fluorescent dyes should be used in the future to increase the accuracy of the identification of BNCs and MN. A further option is to use the MNScore module of the Metafer3 System already in place. In summary, it is possible to use image processing and analysis for the DCA and the CBMN assay to establish automated biodosimetry in the laboratory.

\section{CONCLUSIONS}

Several attempts have been made to increase the speed of DC, CBMN, and $\gamma \mathrm{H} 2 \mathrm{AX}$ assays by using image processing and analysis. During the DCA, variation in the morphologies of metaphase chromosomes and the existence of overlapping or touching chromosomes increases the difficulty of performing DC detection using image analysis. Thus, the use of semi-automatic analysis for automated DCAs that involve operator intervention is considered to be the most appropriate method for obtaining a high degree of accuracy during the automatic identification of DCs. In contrast, due to simpler shapes of BNCs and MN during CBMN assay, many studies have successfully implemented the use of image processing and analysis for automated CBMN assays. However, the longer time required for culture incubations during $\mathrm{CBMN}$ assays compared with DCAs makes automated CBMN assays for biodosimetry less attractive. Future studies on automated CBMN assays should also consider reductions in culture incubation times. Furthermore, for the $\gamma \mathrm{H} 2 \mathrm{AX}$ assay, the replacement of manual analysis with automated analysis based on image processing and analysis provided highly accurate results, timesaving analysis, and the opportunity to extract additional information. Also, because conventional fluorescence microscopes combined with digital cameras are becoming standard equipment in many laboratories and opensource image analysis software designed specifically for biological studies is becoming widely available, the use of image processing and analysis during biodosimetry processes can be performed even in laboratories with standard equipment and limited workforce.

\section{ACKNOWLEDGMENT}

The authors gratefully acknowledge the SAINTEK scholarship program of the Ministry of Research, Technology and Higher Education of the Republic Indonesia. The authors also would like to thank Dr. Radiana Dhewayani Antarianto and Prof. Jeanne Adiwinata Pawitan for the valuable and constructive suggestions to this article.

\section{REFERENCES}

1. A.S. Balajee, M. Escalona, C.J. Iddins et al., Appl. Radiat. Isot. 144 (2019) 111.

2. D. Bakkiam, M. Bhavani, A.A. Anantha Kumar et al., Appl. Radiat. Isot. 99 (2015) 77.

3. I. Karachristou, M. Karakosta, A. Pantelias et al., Mutat. Res. Genet. Toxicol. Environ. Mutagen. 793 (2015) 107.

4. A. Pantelias and G.I. Terzoudi, Mutat. Res. Genet. Toxicol. Environ. Mutagen. 836 (2018) 65.

5. E. Macaeva, M. Mysara, W.H. De Vos et al., Int. J. Radiat. Biol. 95 (2018) 64.

6. U. Oestreicher, D. Endesfelder, M. Gomolka et al., Int. J. Radiat. Biol. 94 (2018) 1017.

7. A.S. Balajee and M.P. Hande, Mutat. Res. Genet. Toxicol. Environ. Mutagen. 836 (2018) 3 .

8. L.A. Beaton-Green, M.A. Rodrigues, S. Lachapelle et al., Methods 112 (2017) 18.

9. G. Gruel, E. Grégoire, S. Lecas et al., Radiat. Res. 179 (2013) 557. 
10. R. Wilkins, M. Rodrigues and L. Beaton-Green, Genome Integr. 8 (2017) 7.

11. P.K. Rogan, Y. Li, A. Wickramasinghe et al., Radiat. Prot. Dosimetry 159 (2014) 95.

12. K. Al-Hadyan, S. Elewisy, B. Moftah et al., 3 Biotech 4 (2014) 635.

13. A.S. Balajee, T. Smith, T. Ryan et al., Radiat. Prot. Dosimetry 182 (2018) 139.

14. H. Dai, J. Feng, H. Bian et al., Dose-Response 16 (2018) 1.

15. P.K. Rogan, Y. Li, R. Wilkins et al., Radiat. Prot. Dosimetry 172 (2016) 217.

16. J. Liu, Y. Li, R. Wilkins et al., F1000Research 6:1396 (2017).

17. B. Shirley, Y. Li, J.H.M. Knoll et al., J. Vis. Exp. 127 (2017) 1.

18. M. François, K. Hochstenbach, W. Leifert et al., Biotechniques 57 (2014) 309.

19. O.V. Lyulko, G. Garty, G. Randers-Pehrson et al., Radiat. Res. 181 (2014) 146.

20. M. Repin, D.J. Brenner and G. Garty, BioRxiv (2019) 620971.

21. M. Repin, S. Pampou, G. Garty et al., Radiat. Res. 191 (2019) 232.

22. C. McQuin, A. Goodman, V. Chernyshev et al., PLoS Biol. 16 (2018) 1.
23. D. Ramadhani and S. Purnami, HAYATI J. Biosci. 20 (2013) 151.

24. S. Zahnreich, A. Ebersberger, B. Kaina et al., Radiat. Res. 183 (2015) 432.

25. A.D. Herbert, A.M. Carr and E. Hoffmann, PLoS One 9 (2014) 1.

26. A. Lapytsko, G. Kollarovic, L. Ivanova et al., BMC Bioinformatics 16 (2015) 17.

27. S. Oeck, N.M. Malewicz, S. Hurst et al., Radiat. Oncol. 10 (2015) 1.

28. S. Oeck, N.M. Malewicz, S. Hurst et al., Radiat. Res. 188 (2017) 114.

29. N. Ruprecht, M.N. Hungerbühler, I.B. Böhm et al., Radiat. Environ. Biophys. 58 (2019) 295.

30. Y. Lusiyanti, Z. Alatas, M. Lubis et al., Atom Indonesia 39 (2013) 124.

31. Y. Lusiyanti, Z. Alatas, M. Syaifudin et al., Genome Integr. 7 (2016) 1.

32. S. Purnami, S. Nurhayati, M. Syaifudin et al., Atom Indonesia 43 (2017) 75.

33. M. Syaifudin, Y. Lusiyanti, S. Purnami et al., Atom Indonesia 43 (2017) 47.

34. Y. Lusiyanti, M. Syaifudin, T. Budiantari et al., Genome Integr. 10 (2019) 1. 